\title{
MANAGEMENT OF SNAKEBITE - CURRENT CONCEPT
}

\author{
Sharma S K ${ }^{1}$, Khanal $B^{1}$, Das B $\mathbf{P}^{1}$, Koirala $\mathbf{S}^{1}$
}

\section{INTRODUCTION}

Snakebite is a medical emergency and a preventable public health hazard in most part of the Nepal. This is a more alarming problem, particularly in terai region. There are 14 species of venomous snakes reported from Nepal, the most common species that are found in tarai region includes found in Cobra, Krait and Green pit Viper etc. ${ }^{1,2}$ Therefore neurotoxic features dominate the clinical picture. The survival of the victim depends on the appropriate first aid measures and immediate transportation to the nearest health center. Identification of the earliest sign of envenomation and immediate institution of anti-snake venom in adequate dosage with other supportive measures are the key to survival of the victim. Development of pre-exposure immunization with vaccines against the snake venom is the newer modalities to be looked ahead in future.

This article aims at reviewing the current concept in the first aid measures and management of snakebite.

\section{FIRST AID}

First aid is carried out as soon as possible after the bite and it is performed by the victim and/or bystander using materials that are readily available and close at hand. However as the most of the available and affordable popular methods are useless or even frankly dangerous, it is essential that only appropriate first aid measures be carried out.

These first aid measures aim $\mathrm{at}^{3}$

1. Retardation of systemic absorption of venom

2. Control of distressing and dangerous early symptoms of envenoming

3. Preserve life and prevent complications before the patient can receive medical care

The recommended first aid measures are a $^{3,4,5,6,7}$

1. Reassurance, as most of the victims of snakebite are terrified and anxious.

2. Immobilization of the bitten limb by using sling or splint. Use of splint with a crepe bandage is very effective, especially in elapid bites. Muscular contraction of bitten limb should be

1. B.P. Koirala Institute of Health Sciences, Dharan, Nepal.

Address for correspondence : Dr. Sanjib Kumar Sharma, MD

Department of Medicine

B. P. Koirala Institute of Health Sciences, Dharan, Nepal

Email: drsanjib@yahoo.com, Fax: 00977-25-20251 
avoided as far as possible, as it will promote venoms return and spread of the venom.

3. Immediate transportation of the victim to the nearest health center, where anti-snake venom (ASV) is available.

4. All harmful and time-wasting treatments should be avoided and strongly discouraged. Certain common practices which need to be avoided are:

? Incision, excision, cauterization and amputation of the bitten digit. These can cause increased bleeding, introduce infection, increased absorption of venom and injury to blood vessels, nerves and tendons and may delay would healing.

? Ingestion of chilies

? Suction of wound by any means can cause tissue necrosis 7,8

? Instillation of chemicals, ice (cryotherapy), herbs and electric shock. These can potentiate local tissue necrosis. ${ }^{4}$

? The role of tourniquet in preventing in preventing systemic envenoming remains controversial. Though animal studies have shown this practice to be beneficial in bite by certain species of snake, in practice it is difficult to judge the appropriate tightness of the tourniquete ${ }^{4.9}$ Moreover the use of tight tourniquet may be extremely painful, can cause ischaemia and the gangrene of the limb (if applied for more than 40 minutes) ${ }^{3}$ Therefore, the use of tourniquet should be discouraged.

5. Pressure immobilization is recommended for bites by neurotoxic elapid snakes, including sea snakes, but should not be used for viper bites because of danger of increasing the local effects of the necrotic venom. ${ }^{3}$

6. The offending snake must not be provoked further by attempts to capture it. However if the snake is already killed, the snake should be carried along with the patient to the hospital.
The snakes should not be handled with bare hands, as even a severed head can bite!

\section{MANAGEMENT AT HEALTH CENTRE}

Rapid clinical assessment, resuscitation and immediate institution of ASV with other supportive care are the key to survival of the victim and to be provided immediately on arrival of the patient.

\section{IMMEDIATE MANAGEMENT}

1. Assessment of vitals and appropriate resusctitation should be the first step in the management.

2. An IV line should be immediately secured and ASV should be made available.

3. Patient should be evaluated for the presence of local and systemic symptoms and signs of envenoming.

4. In the absence of features of envenoming, the patient should be admitted and observed for a minimum period of 24 hours.

5. During the period of observation, the patient should be monitored hourly for local swelling, ptosis, diplopia, respiratory rate and effort, gingival bleed, bleeding from other sites, pulse rate and rhythm, blood pressure, urine output, cola colored urine, level of consciousness and any other signs of envenoming.

6. Certain clinical situations demand urgent resuscitation ${ }^{3}$ e.g.

? Profound hypotension and shock

? Respiratory failure

? Sudden deterioration or rapid developments of severe systemic envenomation following release of tourniquet or compression bandage.

? Cardiac arrest due to rhabdomyolysis

? Late complication of envenomation such as renal failure and sepsis. 


\begin{abstract}
ANTI-SNAKE VENOM THERAPY
Before introduction of $\mathrm{ASV}$ the treatment of snakebite was mainly symptomatic. Viper bite with coagulopathy was treated with fresh blood transfusions and elapidae bite with neurotoxicity with injection neostigmine and respiratory support. Availability of ASV has opened up a new vista to treatment of such patients.
\end{abstract}

Anti-snake venom is a whole serum or enzymerefined immunoglobulin of animals, usually horses or sheep. It is the only specific treatment with proven efficacy against many of the lethal and damaging effects of the snake venom. In the management of snakebite, the most important clinical decision is whether or not to administer ASV. The use of ASV should be discrete and rational, as only a minority of snake-bitten patients needs it. Besides, it may produce severe untoward reactions and cause sensitization. ASV is expensive and is often in short supply.

\section{PREPARATIONS OF ASV}

i. Polyvalent ASV

ii. Monovalent ASV

\section{a. Polyvalent ASV}

Polyvalent ASV contains specific antibodies against snake venom of more than two snake species. The concentrations of specific antibodies to each species is low, as compared to monovalent anti-snake venom. In Nepal, only polyvalent ASV is available. In endemic countries, several species may produce identical signs, patients most often do not see the snake, and even if they see, not all of them can identical signs, patients most often do not see the snake, and even if they see, not all of them can identify the species accurately and killed dead snakes for identification are brought very infrequently. Under such a situation, it is safer to treat the patients with polyvalent ASV. Antibodies raised against the venom of one species may have cross-neutralising activity against other venoms, usually from closely related species. This is known as paraspecific activity. ${ }^{3}$ For example, the manufacturers of Haffkine polyvalent anti-snake venom serum claim that this antivenom also neutralizes venom of two Trimersurus species. ${ }^{3}$

\section{b. Monovalent ASV}

Since its introduction, ASV has undergone several modifications and improvement all over the world. Now a days highly specific and potent monovalent anti-serum against each species of the venomous snakes is available. If the biting species is known or can reliably be deduced, the appropriate monovalent anti-snake venom is indicated.

\section{FORMS OF ASV}

ASV in the form of concentrated and purified hyper-immunized horse plasma is available in two forms:

i. Liquid form (Central Research Institute, Kasauli)

ii. Lyophilized form (Haffkine Biopharmaceutical Corporation Ltd, Mumbai)

While the liquid form requires storage at $4^{0} \mathrm{C}$ and has relatively shorter half life, the lyophilized form, which is practically devoid of moisture content, retains its potency on storage at below $8^{\circ} \mathrm{C}$ or room temperature (cool, dark room) for more than 5 years. ${ }^{4}$ Opaque solutions should not be administered as precipitation of protein indicates loss of activity and an increased risk of reaction. Each $\mathrm{ml}$ of reconstituted polyyalent ASV serum neutralizes not less than the following quantities of standard venom when tested in white mice: 


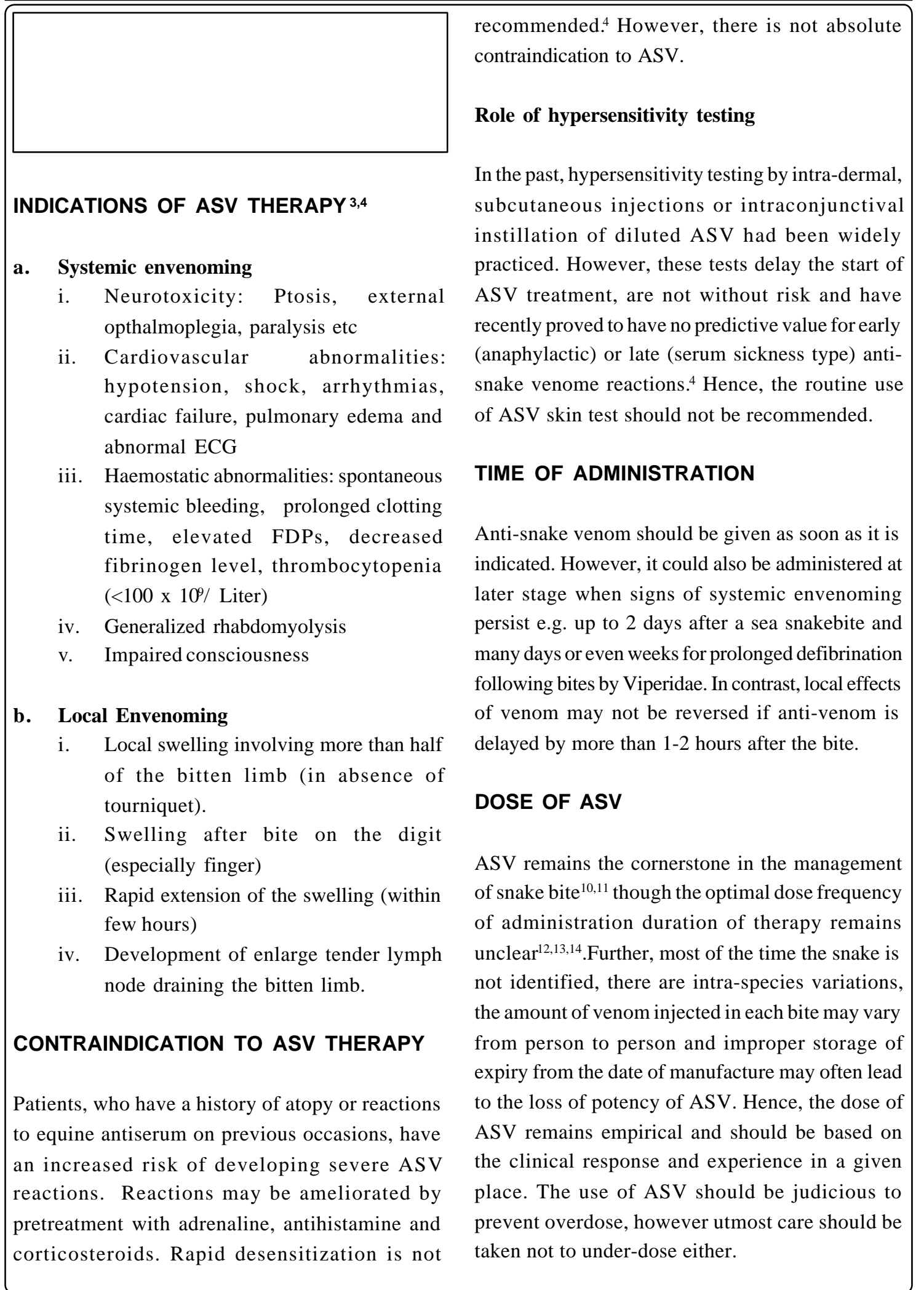


Snakes inject the same dose of venom into children and adults. Children must therefore be given exactly the same dose of antivenom as adults. ${ }^{3,4}$

\section{a. Elapid bite}

The recommended initial dose of ASV in case of elapid bite with systemic envenomation is $100 \mathrm{ml}$. Larger doses may be required for the large sized snake. The dose may be reduced to half in cases related to clinical features of local envenomation. The dose may be repeated after one hour if the clinical signs detoriate $50-100 \mathrm{ml}$ of ASV is repeated thereafter, every $4-6$ hours until nerotoxic signs disappear. In case of King cobra bite $100 \mathrm{ml}$ of monovalent ASV used as initial dose, if available. ${ }^{6}$ The average dose requirement of ASV in cases of krait bite is reported to vary from $90 \mathrm{ml}$ to $430 \mathrm{ml}$ in various studies. ${ }^{15,16,17,18}$ Gonthavorn ${ }^{19}$ reported the use of $1150 \mathrm{ml}$ of ASV in a King cobra bite.

\section{b. Viper bite}

Several studies have shown variation in the average dose required for the treatment of viper bite. ${ }^{12,20}$ One of the probable factors attributed to this might be intra-species variation. Hence, the dosage requirement for different geographic areas should be locally determined by clinical studies. One of the suggested recommendation is as follows. ${ }^{12}$

Initial dose - 20 to $100 \mathrm{ml}$ (according to regional requirement)

Repeat dose - 20 to $50 \mathrm{ml}$ every 4-6 hours until clotting time returns to normal

Recurrence of coagulation dysfunction - 20 to $50 \mathrm{ml}$

\section{ROUTES OF ADMINISTRATION}

Anti-snake venom may be administered through the following routes:

a. Intravenous

b. Intra-muscular

\section{a. Intravenous route}

The intravenous route is the most effective and the preferred one for ASV administration. There are two modes of administration of ASV through the intravenous route. Their advantages and disadvantages are discussed below.

\section{i. Single large bolus:}

This was the only method practiced before the $\mathrm{t}$ has been abandoned since ASV has limited halflife and thus is not capable of preventing late envenomation. ${ }^{4}$

\section{ii. Intermittent (small) bolus doses (Intravenous "push" injection)}

This is the most widely practiced in all endemic areas. ASV is given by slow intravenous injection (not more than $2 \mathrm{ml} / \mathrm{minute}$ ), usually every six hours until signs of systemic envenoming disappear. This mode of administration of ASV gives ample coverage against late envenoming. Since the maximum clinical effect on the restoration of normal coagulation occurs four hours after ASV administration, this practice gives scope for adequately titrating and controlling dose of ASV for residual correction of coagulation defect (the subsequent dose requirement being low) However, it may take longer time to restore normal coagulation in certain instances. Prevention of misuse of larger doses of ASV in undeserving cases and better control of coagulation dysfunction have made this the most popular form of delivery of ASV.

\section{iii. Continuous intravenous infusion with intermittent bolus}

Practice of such delivery of ASV may be the best method to better titration the dose of ASV and is likely to further reduce the total dose of ASV vis- 
à-vis the previous method. But slower correction of preventable life-threatening bleeding may prove costly. It is also likely that total requirement of ASV by second or third method may be same in most of the instances. The difference in the incidence of severity of anti-venom reactions in patients treated by either methods were also not reported.,21

\section{b. Intramuscular route}

In the absence of adequate expertise to administered intravenous ASV this may be given by deep intramuscular injection (e.g. in the anterior thighs but not in the gluteal region) followed by massage to promote absorption. ASV is large molecules and absorption is very slow after intramuscular injection. ${ }^{3,4}$ This also accounts for requirement of a higher dose and formation of hematoma following intramuscular injection.

\section{REACTIONS TO ASV}

Three types of reactions may complicate ASV administration:

i. Early (anaphylactic) reactions

ii. Pyrogenic reactions

iii. Late reactions

\section{i. Early (anaphylactic) reactions}

Hypersensitivity reactions to equine serum proteins could not be detected reliably either by skin testing or radiolallergosorbent tests (RAST). ${ }^{3,21}$ ASV activates complement in vitro, ${ }^{22}$ while the clinically similar reactions to homologous serum are associated with complement activation and immune complex formation in vivo. The complement system is probably activated by aggregates of $\mathrm{IgG}$.

The reactions usually develop within 10-20 min (IV bolus) or 30 min-3 hrs (IV infusions) of starting the ASV. The symptoms are initially in form of restlessness, cough, itching, nausea, vomiting, feeling of heat, tachycardia and later urticaria, generalized pruritis, fever, tachycardia, autonomic symptoms, hypotension, airflow obstruction, angioedema predominate the picture. Unless patients are watched carefully for 3 hours after treatment, mild reactions may be missed and deaths may be attributed to the envenoming. The reported incidence varies from 3 to $54 \%$ appears to increase with the dose and decrease when refined ASV is used. The incidence is found to be lower when administration is by intra-muscular rather than intravenous injection.

Early reactions respond readily to adrenaline given by an intra-muscular route (into the deltoid muscle or the upper lateral thigh) in an initial dose of 0.5 $\mathrm{mg}$ for adults and $0.01 \mathrm{mg} / \mathrm{kg}$ body weight for children at the first sign of reaction. ${ }^{3}$ The dose can be repeated every 5 to 10 minutes. Anti-H1 antihistaminics such as chlopheniramine maleate (adults $10 \mathrm{mg}$, children $0.2 \mathrm{mg} / \mathrm{kg}$ by intravenous injection over a few minutes) should be given followed by intravenous hydrocortisone (adults 100 $\mathrm{mg}$, children $2 \mathrm{mg} / \mathrm{kg}$ body weight). The corticosteroid is unlikely to act for several hours, but may prevent recurrent anaphylaxis. ${ }^{3}$

\section{ii. Pyrogenic reactions}

It results from contamination of the anti-venom by endotoxin like compounds. High fever develops 1 -2 hours after treatment and is associated with rigors, followed by vasodilation and a fall in blood pressure. Febrile convulsions may be precipitated in children. It is treated with hydrotherapy and antipyretic drugs such as paracetamol.

\section{iii. Late reactions}

It develops 5 - 24 (mean 7) days after treatment. The higher the dose of anti-venom the higher the 
incidence of these reactions and the speed of their development. Symptoms include fever, urticaria, subcutaneous and peri-articular swelling, polyarthritis, lymphadenopathy, mononeuritis multiplex, albuminuria and rarely encephalopathy. This is an immune complex disease, which responds to anti-histaminics in mild cases while corticosteroids (prednisolone $5 \mathrm{mg}$ four times a day for 5 days) would be required in severe cases.

\section{SUPPORTIVE MEASURES Cholinesterase}

The neuro-paralytic symptoms in elapid bite are due to the offending toxins, cobratoxin and alphabungarotoxin, those produce the neuromuscular block by acting on the post-synaptic junction of the motor end plate. This blockade can be reversed by neostigmine. ${ }^{23}$ A clinical study showed a survival of over $95 \%$ in cases treated with neostigmine and ASV as compared to $22.6 \%$ when treated with ASV alone. ${ }^{24}$ This study also showed a significantly faster pace of recovery in the neostigmine group. According to this study, best results can be obtained by starting neostigmine as soon as the neurological signs appear and it is essential to continue neostigmine till the complete neurological recovery is made, as otherwise premature discontinuation may lead to relapse of neuro-paralysis. It is essential to combine neostigmine with a preceding intravenous administration of anti-cholinergic drug like atropine sulphate, to ward off its side effects on bronchial secretions and cardiac rate. It is preferable to do "Tensilon test" if available prior to giving neostigmine.

\section{Recommended treatment protocol $3,23,24$}

Following the routine anti-venom doses, neostigmine is given only in those cases that present with definite signs of neuro-paralysis. Intravenous neostigmine, $0.5 \mathrm{mg}$ is given at half hourly interval for five injections. This is followed by repeating the same dose at increasing intervals of 2 to 12 hours according to the state of neurological recovery. Each dose of neostigmine is preceded by an intravenous injection of $0.6 \mathrm{mg}$ atropine sulphate to ensure a rise in the pulse rate by 20 beats per minute. The injections can be conveniently given through the saline infusion line. Patients who are able to swallow tablets may be maintained on atropine $0.6 \mathrm{mg}$ twice each day, neostigmine 15 $\mathrm{mg}$ four times each day or pyridostigmine $60 \mathrm{mg}$ four times each day (initial adult doses).

Neostigmine-atropine in the recommended dosage schedule has been found to be completely free from any untoward effect and is considered fully safe. The encouraging results with the treatment suggest that since the cobra-neurotoxin and the alphabungarotoxin form the major toxins in overwhelming majority of elapid envenoming in Nepal, this treatment should be employed as a routine supplementary treatment to anti-venom therapy in all cases of neurotoxic snakebite.

\section{Artificial Ventilation}

Artificial ventilation was first suggested for neurotoxic envenoming more than one hundred years ago but patients continue to die for lack of this simple procedure. Neuro-paralytic effects are fully reversible, however, it may take few days to several weeks. Once there is loss of gag \& cough reflexes or respiratory distress suggesting bulbar and respiratory paralysis, endotracheal intubation or tracheostomy, using cuffed tubes, is needed. The patient can be ventilated manually with an anaesthetic or Ambu bag or, preferably, with a mechanical ventilator. Even complete recovery of neuromasculer symptoms and respiratory depression within 36 - 72 hours artificial respiration is absence of AIV is reported ${ }^{14}$. 
Thus, non- availabity of ASV should not deter a physician from successfully treating a patient of snakebite with neuro-paralytic symptoms. Further, combination of aforementioned measures may reduce the total requirement of ASV. However, if ASV is available, other measures cannot be uses altogether as substitute to ASV.

\section{HAEMOSTATIC DISTURBANCES}

Once specific anti-venom has been given to neutralize venom pro-coagulants, restoration of coagulability and platelet function may be accelerated by giving fresh whole blood, fresh frozen plasma, cryoprecipitates containing fibrinogen, factor VIII, fibronectin, and some factors V and XIII or platelet concentrates.

\section{Hypotension and Shock}

These usually result form hypovolemia and should be treated by infusing a plasma expander, preferably fresh whole blood or, failing that, fresh frozen plasma. Central venous pressure or pulmonary arterial catheter monitoring is the safest way to control volume replacement. Hypotensive patients would also respond to dopamine infusion. Other causes of hypotension are anaphylacsis, vasodilatation, cardiac abnormality, and respiratory failure etc.

\section{Local Infection}

Infection at the site of the bite should be prevented with penicillin or erythromycin (or by an antimicrobial effective against the bacterial flora of the buccal cavity and venoms of local snakes) and a booster dose of tetanus toxoid should be given., ${ }^{3,25}$ An aminoglycoside such as gentamicin should be added if the wound has been tampered with or there is evidence of local necrosis. Bullae are best left alone. Snake bitten limbs should be nursed in the most comfortable position. Necrotic tissue should be excised as soon as possible and denuded area covered with split skin grafts.

\section{Intracompartmental Syndromes,}

Swelling of muscles within tight fascial compartments may raise the tissue pressure to such an extent that perfusion is impaired and thus causing ischaemic damage. The signs of an intracompartmental syndrome include excessive pain, weakness of the compartmental muscles and pain when they are passively stretched, hypoaesthesia of areas of skin supplied by nerves running through the compartment and obvious tenseness of the compartment. Palpation of peripheral pulses or their detection by Doppler ultrasound does not exclude intracompartmental ischaemia. Fasciotomy must not be attempted before blood coagulability has been restored by adequate doses of specific antivenom, and by transfusion of fresh whole blood or clotting factors. Corticosteroids, heparin, antifibrinolytic agents such aprotinin (Trasylol) and ?-aminocaproic acid, antihistamine, trypsin and a variety of traditional herbal remedies have been used and advocated by different people. However, many of them are potentially harmful and none have been proved to be effective.

\section{REHABILITATION}

Bitten part may develop contracture and deformity. To avoid this limbs are kept in functional position and physiotherapy should be given.

\section{IMMUNIZATION AGAINSTENVENOMING}

An exciting area in the prevention of mortality and morbidity due to snake envenoming is the production of vaccines that can induce protective levels of antibodies against lethal venom components by pre-exposure immunization in high 
risk populations. Trials using vaccine against $\mathrm{T}$. flavoviraidi conducted in Ryuku and Amami Islands of Japan have had inconclusive results. ${ }^{27}$ Elsewhere, there has been some progress in producing venoids to protect against Russell's viper bite. In this case, the rapid development of renal damage, irreversible by antivenom, is a strong argument for pre-exposure prophylaxis. ${ }^{28}$ The production and modification of venom antigen by genetic engineering is an exciting new development that could lead to the production of snake venom vaccines. ${ }^{29}$

\section{REFERENCES}

1. B hetwal B B, O 'S hea M, W arelI D A.S nakeand snakebite in N epal. T rop D oc 1998; 28: 193-5.

2. J oshi D D. E pidemiological surveillance on snakebite and mortality in $N$ epal. I ournal of $N$ epal M edical A ssociation 1982; 20: 22 - 30.

3. W arelID A W W O /SEA R O G uidelines for "T he clinical management of snake bites in the the $S$ outheast A sian region".S outheast A sian J T rop $M$ ed $P$ ublic $H$ ealth 1999;30(S upp):1-85.

4. W arrell D A.A nimal T oxins.I n: C ook G (editor) $M$ anson's $T$ ropical $D$ iseases. $20^{\text {th }}$ e. (E L B S ), L ondon, W B S aunders, 1996; 468-515.

5. P aul V K. A nimal and insect bites. I n:M eherban $S$ ingh.E d.M edical $E$ mergencies in $C$ hildren.2 ${ }^{\text {nd }}$ edition.S agar publication,N ew D el hi 1993.

6. $R$ ussel FE .S nakeV enom P oisoning.J B L ippincott C ompany.P hiladel phia.1980 pp291-350.

7. $\quad H$ ardy $D L . A$ review of first aid measures for pit viper bite in $\mathrm{N}$ orth $\mathrm{A}$ merica with an appraisal of $E$ xtractor suction and stun gun electroshock. I n: $C$ ampbell J a, B rodie E D (editors) B iology of the pit V ipers. T yler, T X ; S elva, 1992; 405-14

8. G ellert GA.S nake venom and insect venom extractor :an unproved therapy.N $\mathrm{E} n \mathrm{ng} \mathrm{M}$ ed $1993 ; 328: 1322$.

9. W arrell D A. T he global problem of snakebite: its prevention and treatment. I $\mathrm{n}$ : $\mathrm{G}$ opalakrishnakone
$P$ and $T$ an $C K$ (editors) $R$ ecent $A$ dvances in $T$ oxicology $R$ esearch. $N$ ational $U$ niversity of S ingapore, 1992;1:121-53.

10. Thomas $P P, J$ acob J. R andomised trial of antivenom in snake envenomation with prolonged clottingtime. B r M ed J 1985; 291: 177-8.

11. S eiler $G 3^{\text {rd }}, S$ agerman $S D, G$ eller $R$ J , E Idridge J $C$, F leming $L \quad L$. V enomous snakebite; $C$ urrent concept of treatment. 0 rthopedics 1994; 17; $707-14$.

12. D utta $T K, G$ hotekar $L H, R$ ational use of anti-snake venom (A SV ).I n: D as A K (editor) M edicineU pdate $1998 \mathrm{~T}$ he A ssoc of $P$ hysicians of I ndia $1998 ; 8: 760-5$.

13. $R$ eid $H A$, T heakston $R D, T$ he management of snakebite. B ull W orld H ealth 0 rg 1983; 61(6): $885-95$.

14. P ochunugul $C, L$ imthongkul $S, S$ itpriya $V, B$ enyajati $C$. M anagement of $C$ obrabite by artificial respiration and supportive therapy. I $M$ ed $A$ Ssoc T hai 1994; 77: $161-64$.

15. T heastone $R D, P$ hilips $R E, W$ arell $D A$, $G$ alegedera $Y$ et al. E nvenoming by the common krait( $B$ angarus caeruleus) and $S$ ri $L$ ankan cobra ( $N$ aja naja maja): efficacy and complication of therapy with $H$ affkine antivenom. $T$ rans $R$ oy $S$ oc T rop $M$ ed H yg 1990; 84: 301 - 8 .

16. S aini $R K, S$ ingh $S, S$ harma $S, R$ amphal $V, M$ anhas A S, G upta V K . S nakebite poisoning presenting as early morning paralytic syndrome in jhhuggi dwellers. I A ssoc P hysician of I ndia 1986; 34: $415-417$.

17. $H$ ati $A$ k, $S$ aha $S G$, banerjee $D, P$ anda $D$. C linical feature of poisoning by common kraits and treatment with polyval ent antivenom. $T$ he $S$ nake 1988; 20: 140 - 43.

18. F ernando $P, D$ ias $S, A$ case report. I ndian krait bite poisoning. C yelon medical J ournal 1982; 27: $39-41$.

19. G onthavorn S. A case of $K$ ing cobra bite. $T$ oxicon 1971; 9: 293 - 94.

20. V ijeth SR. S tudy of clinical and haematological profile of snakebite and its management. 
$D$ ossertation submitted to $P$ ondicherry $U$ niversity towards completion M D D egree course, 1994.

21. M alasit $P, W$ arrel I D A, C hanthavanich $P$. $P$ rediction, prevention and mechanism of early (anaphylactic) anti-venom reactions in victims of snakebites. B r M ed J 1986;292: 17-20.

22. S utherland SK. S erum reactions. A $n$ analysis of commercial antivenoms and the possible role of anticomplementary activity in de- novo reactions to antivenoms and antitoxins. M ed I A ust $1977 ; 1: 613-5$.

23. B enerjee $R N$. P oisonous snakes of I ndia, their venoms, symptomatology and treatment of envenomation. I $\mathrm{n}$ : A huja M M S (editor) $P$ rogress in clinical medicine in I ndia. A rnold $\mathrm{H}$ einemann P ublishers. 1978;2:136-79.

24. G eorge W att, $R$ D G T heakstone, $C$ urtis $G$ et al. $P$ ositive response to edrophonium in patients with neurotoxic envenoming by cobras ( $N$ aja $\mathrm{N}$ aja $P$ hilippinesis): a placebo controlled study. N E ng J $M$ ed 1986; 315: 1444 - 8 .
25. T heakston $R$ D G , P hillips $R E, L$ ooareesuwan $S$, $E$ cheverria $P, M$ akin $T, W$ arrell D A . B acteriological studies of the venom and mouth cavities of wild $M$ alayan pit viper in southern $T$ hailand. $T$ rans $R$ S oc T rop M ed H yg 1990;84:875-9.

26. M atsen FA. C ompartmental syndromes. N ew Y ork: $G$ rune and $S$ tratton 1980; 162.

27. S awai $Y, V$ accination against snakebite poisoning. I $n$ : $L$ ee $C Y$ (editor) $S$ nake venoms. $H$ andbook of Experimental $P$ harmacology. $B$ erlin: S pringer, 1979: 881-97.

28. M yint- $L$ win, $W$ arrell $D A, P$ hilips $R E$, etal. $B$ itesby $R$ ussell's viper ( $V$ ipera resselli siamensis) in B urma: haemostatic, vascular and renal disturbances and response to treatment. $L$ ancet $1985 ; 2: 1259-64$.

29. M enez $A$. I mmunology of snake toxins. I n: $H$ arvey $A \quad L$ (editor) $S$ nakeT oxins: I nternational E ncyclopedia of $P$ harmacology and $T$ herapeutics $S$ ec. 134. N ew Y ork, $\mathrm{P}$ ergamon press, 1991; 35-90.

$$
\text { L } 20
$$

